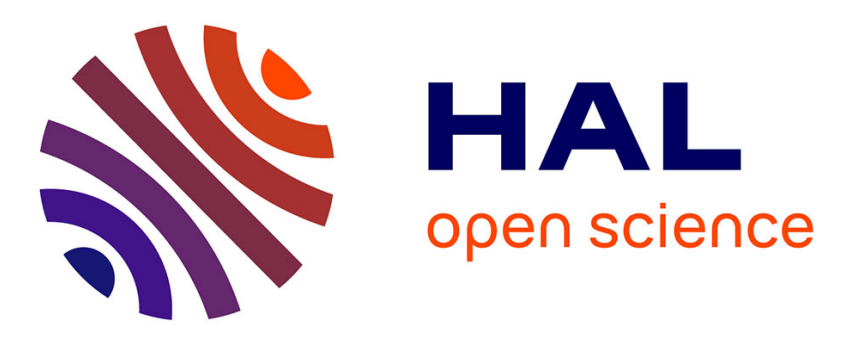

\title{
Ein Blick von außen: Die Frankreichstrategie des Saarlandes aus lothringischer Perspektive
}

\author{
Reiner Marcowitz
}

\section{To cite this version:}

Reiner Marcowitz. Ein Blick von außen: Die Frankreichstrategie des Saarlandes aus lothringischer Perspektive. Hans-Jürgen Lüsebrink; Claudia Polzin-Haumann; Christoph Vatter. "Alles Frankreich oder was ¿̆" - Die saarländische Frankreichstrategie im europäischen Kontext / "La France à toutes les sauces ¿' - La 'Stratégie France' de la Sarre dans le contexte européen, 15, transcript, pp.83-94, 2017, Jahrbuch des Frankreichzentrums, 10.14361/9783839437551 . hal-02904720

\section{HAL Id: hal-02904720 \\ https://hal.univ-lorraine.fr/hal-02904720}

Submitted on 22 Jul 2020

HAL is a multi-disciplinary open access archive for the deposit and dissemination of scientific research documents, whether they are published or not. The documents may come from teaching and research institutions in France or abroad, or from public or private research centers.
L'archive ouverte pluridisciplinaire HAL, est destinée au dépôt et à la diffusion de documents scientifiques de niveau recherche, publiés ou non, émanant des établissements d'enseignement et de recherche français ou étrangers, des laboratoires publics ou privés. 
Reiner Marcowitz

\section{Ein Blick von außen:}

\section{Die Frankreichstrategie des Saarlandes aus lothringischer Perspektive}

Cet article traite, tout d'abord, de la réaction française et plus spécifiquement lorraine majoritairement positive à la 'Stratégie France' de la Sarre pour autant qu'elle se reflète dans la presse et dans les prises de positions des différents acteurs de la société civile. Ensuite, l'article analyse la genèse et le contenu de la 'Stratégie Allemagne' de la Lorraine qui se trouve être une réponse directe à son équivalent sarrois. Enfin, l'article développe différents scénarios sur l'évolution future et sur l'éventuelle réussite ou bien l'échec de ces deux projets complémentaires. Il attire également l'attention sur le fait que leur réalisation est importante tant au niveau régional qu'au niveau (inter)national afin d'agir contre le désintérêt croissant dans les sociétés allemande et française pour le voisin respectif, et afin de rendre au partenariat franco-allemand son importance qui est non seulement historique, mais également indispensable pour la réussite de l'Union Européenne.

Das Ziel dieses Beitrags ist es, Einblicke in die lothringische Sicht auf die saarländische Frankreichstrategie zu geben und über das Echo zu berichten, das diese Initiative jenseits der Grenze gefunden hat. ${ }^{1}$ Dies geschieht in drei Schritten: Zunächst werden die unmittelbaren Reaktionen auf das saarländische Projekt in den lothringischen bzw. französischen Medien sowie seitens einzelner zivilgesellschaftlicher Institutionen untersucht (1.). In einem zweiten Schritt werden dann die Überlegungen zu einer lothringischen Deutschlandstrategie vorgestellt, die gewissermaßen die kongeniale Antwort auf die saarländische Initiative darstellen (2.). Schließlich werden die Zukunftsaussichten beider Strategien erörtert (3.).

1 Der Autor hat im Rahmen seiner beruflichen Tätigkeit im Frühjahr/Sommer 2015 eine Arbeitsgruppe zum Thema Hochschule und Forschung geleitet, die für diesen Bereich einen Beitrag zur Ausarbeitung einer Deutschlandstrategie Lothringens geleistet hat. Die betreffende Arbeit, die im vorliegenden Beitrag noch näher vorgestellt wird, wurde in völliger Unabhängigkeit geleistet. Zudem ist sie zumindest für den Augenblick abgeschlossen und auch insofern gibt der vorliegende Aufsatz nur die persönliche Meinung des Verfassers wieder. 


\section{Die saarländische Frankreichstrategie und ihr lothringisches Echo}

Wie letztlich nicht anders zu erwarten, war das Medienecho auf die Ankündigung der saarländischen Frankreichstrategie Anfang 2014 in Frankreich im Allgemeinen und in Lothringen im Besonderen positiv. Das saarländische Bekenntnis zur von den Journalisten gerne zitierten Sprache Molières entsprach, ja schmeichelte dem nationalen französischen Selbstverständnis, als Kulturnation gerade auch eine sprachliche mission civilisatrice zu haben. ${ }^{2}$ Im Falle Lothringen kam noch die geographische und geschichtliche Nähe hinzu: ${ }^{3}$ Die Grenzen und die nationale Zugehörigkeit waren hier jahrhundertelang fließend und wechselten einander ab. Entsprechend sind gerade diese beiden Regionen in vielfältiger Weise besonders miteinander verflochten. Folglich war auch hier das Medienecho auf die Ankündigung der saarländischen Frankreichstrategie sehr positiv: Zunächst wurde die Originalität der saarländischen Initiative und deren Beitrag zur deutsch-französischen Zusammenarbeit auch in der nächsten, der etwas missverständlich sogenannten „Generation Elysée“ gelobt. ${ }^{4}$ Dies ging sodann einher mit der Einsicht in die ökonomischen Vorteile, die die forcierte Frankreichorientierung des deutschen Nachbarn gerade auch der eigenen Region und seinen Berufspendlern eröffnete. Allerdings wurde in einigen Artikeln auch bereits auf die Probleme hingewiesen, die die saarländische Landesregierung zu ihrer Initiative bewogen habe: der Bevölkerungsrückgang des ohnehin schon bevölkerungsschwachen und kleinen Bundeslandes, die hohe Verschuldung des 'deutschen Griechenlands' („Grèce de l'Allemagne“"5), der deshalb zumindest mittel- und langfristig drohende Verlust der Autonomie im Rahmen einer etwaigen Fusion mit einem der angrenzenden Bundesländer. Vor diesem Hintergrund wurde von einigen Journalisten ebenfalls die Frage nach der Finanzierbarkeit des Projekts gestellt. Ebenso wurde zumindest vereinzelt darauf hingewiesen, dass v. a. bei saarländischen Eltern Widerstände gegen das erzwungene Erlernen der französischen Sprache bestünden, zumal wenn dieses auf Kosten des Englischunterrichts ginge.

2 Vgl. Delorme, Florian: La bataille des langues en Europe (2/4) - De l'école au travail, enjeux linguistiques en zones transfrontalières, in: France Culture Radio, 18.03.2014, http:// www.franceculture.fr/emissions/culturesmonde/la-bataille-des-langues-en-europe-24-delecole-au-travail-enjeux (14.04.2016).

3 Zur Geschichte Lothringens vgl. Parisse, Michel (Hg.): Lotbringen. Geschichte eines Grenzlandes, Saarbrücken: Saarbrücker Druckerei u. Verl., 1983; Bastien, René: Histoire de la Lorraine, Metz: Editions Serpenoise, 1993.

4 Vgl. Knoepffler, Christian: Frankreich über alles, in: Le Républicain Lorrain, 05.02.2014, S. 7.

5 O. A.: Pour survivre, la Sarre parie sur le parler français, in: Le Télégramme, 03.03.2014, S. 1. 
Seitens der Zivilgesellschaft reagierte der lothringische Wirtschafts-, Sozialund Umweltrat, eine Einrichtung des Regionalrats von Lothringen, sicher am enthusiastischsten: Er lud den saarländischen Europa- und Finanzminister Stephan Toscani im Mai 2014 sogar ein, die Strategie vor Ort den eigenen Delegierten zu präsentieren. ${ }^{6}$ Zudem arbeitete er im Frühjahr 2014 eine ausführliche Stellungnahme aus, die bereits den programmatischen Untertitel trug: 'eine Gelegenheit, die ergriffen werden muss, eine Verpflichtung zu handeln'?. Anknüpfend an eine große Studie, die der Conseil Economique, Social et Environnemental (CESEL) bereits im November 2011 unter dem ebenfalls ,sprechenden' Titel 'Deutschland, ein unersetzlicher Partner Lothringens' vorgestellt hatte, ${ }^{8}$ erinnerte er an die Bedeutung Deutschlands und speziell des Saarlandes für die lothringische Wirtschaft: So pendeln trotz eines seit 2002 spürbaren Rückgangs um 5000 Menschen aktuell immer noch fast 20000 Lothringer (Stand 2013) jeden Tag zur Arbeit nach Deutschland, zudem hängen weitere 42000 Arbeitsplätze in Lothringen an deutschen Investitionen in der Region sowie am Export lothringischer Unternehmen nach Deutschland. ${ }^{\text {? }}$

Vor diesem Hintergrund begrüßte der CESEL in seiner Stellungnahme vom Frühjahr 2014 einhellig die Frankreichstrategie des Saarlandes: Diese nutze die besondere Kompetenz des Saarlandes und gebe diesem gleichzeitig ein Alleinstellungsmerkmal als 'Versuchslabor' für neue Formen grenzüberschreitender Zusammenarbeit. Folglich könne das Projekt ein 'Sprungbrett' sein, 'um diesen Status eines Versuchslabors zu bekräftigen'10. Gleichzeitig unterbreitete der CESEL acht Vorschläge für konkrete Maßnahmen von lothringischer Seite, die die saarländische Frankreichstrategie unterstützen sollten. ${ }^{11}$ Sie kulminierten in der Forderung nach einer lothringischen Deutschlandstrategie, komplementär zum Frankreichprojekt des Saarlandes. Bei der entsprechenden Umsetzung der beiden Strategien sollte zudem auf die Integration von Vertretern des Partnerlandes geachtet werden, wenn möglich

6 Vgl. Conseil Economique, Social et Environnemental de Lorraine (CESEL): A la Une. Stratégie France: le CESEL présente 8 pistes d'actions côté lorrain, 20.05.2014, http:// www.ceselorraine.eu/fr/strategie-france-le-cesel-presente-8-pistes-d-actions-cote-lorrain/ a-la-une-du-cese-lorraine.html (14.04.2016).

7 Vgl. CESEL: Avis du CESEL sur la Stratégie France de la Sarre. Une opportunité à saisir, une obligation d'agir. Avis adopté à l'unanimité en Séance Plénière du 16 mai 2014, http:// www.ceselorraine.eu/img_base/ged_v2/278/2014-cesel-avis-strategie-france-sarre.pdf (14.04.2016).

8 Vgl. CESEL: L'Allemagne, partenaire incontournable de la Lorraine. Projet. Séance Plénière du 18 octobre 2011, http://www.ceselorraine.eu/UserFiles/File/ressources/ rapports/2011/2011-cesel-rapport-allemagne.pdf (14.04.2016).

9 Vgl. CESEL: Avis du CESEL sur la Stratégie France, S. 4 f.

10 Vgl. CESEL: Avis du CESEL sur la Stratégie France, S. 8.

11 Vgl. CESEL: Avis du CESEL sur la Stratégie France, S. 9-12. 
sogar die Bildung spiegelbildlicher Einrichtungen angestrebt werden, analog dem Frankreichzentrum der Universität des Saarlandes und seiner jüngeren lothringischen ,Schwestereinrichtung, dem Centre franco-allemand de Lorraine (CFALOR).

Mit dem letzten Punkt wurde bereits an die Vorbildrolle der Universität des Saarlandes und der Universität Lothringen für die regionale Kooperation erinnert. Auf diese berief sich auch die Leitung der Universität Lothringen in ihrem Kommentar zur Frankreichstrategie, um die die saarländische Landesregierung sie gebeten hatte. ${ }^{12}$ Tatsächlich bietet die lothringische Universität allein 47 Studiengänge mit Deutschlandbezug an, darunter 24 in Kooperation mit der Hochschule für Technik und Wirtschaft in Saarbrücken oder mit der Universität des Saarlandes. Vor diesem Hintergrund resümierte die Stellungnahme der Universität, „dass Deutschland das Zielland Nr. 1 der Universität ${ }^{\text {"13 }}$ sei, weswegen Ende 2012 auch das CFALOR gegründet worden sei. Insofern wurden auch die konkreten Maßnahmen und insbesondere die sprachpolitische Ambition der saarländischen Frankreichstrategie begrüßt. Folgerichtig formulierte auch die Universitätsleitung den Wunsch, dass Lothringen analog zum saarländischen Projekt eine eigene Deutschlandstrategie entwickeln solle.

Die Stellungnahmen von CESEL und der Universität zur saarländischen Frankreichstrategie verwiesen also, wie im Übrigen auch schon der eine oder andere Pressekommentar, auf die geradezu logisch anmutende Konsequenz einer komplementären lothringischen Deutschlandstrategie. Diesen Ball nahm die Politik dann auch tatsächlich Ende 2014 auf.

\section{Die lothringische Deutschlandstrategie als Pendant zur saarländischen Frankreichstrategie}

Im September 2014 erteilte der Präsident der Region Lothringen (CRL), JeanPierre Masseret, dem Präsidenten des Departements Meurthe et Moselle (CD 54), Jean-Matthieu Klein, den Auftrag, in Kooperation mit Patrick Weiten, dem Präsidenten des Conseil départemental Moselle (CD 57), eine Deutschlandstrategie Lothringens auszuarbeiten. ${ }^{14}$ Eine Pilotgruppe von Vertretern der verschiedenen Gebietskörperschaften, Berufsverbänden, der Universität und dem französischen Generalkonsulat beschloss anschließend im

12 Vgl. Stellungnahme der Universität Lothringen zur Frankreichstrategie des Saarlandes vom 02.06.2014, Kopie im persönlichen Besitz des Autors.

13 Stellungnahme der Universität Lothringen zur Frankreichstrategie des Saarlandes.

14 Vgl. Stratégie Allemagne de la Lorraine. Rapport final. Synthèse et propositions juin 2015, 2015, http://metz.fr/pages/conseil_municipal/seances/cm151029/doc/5_d1445930773387. $\operatorname{pdf}(14.04 .2016)$, S. 2. 
Frühjahr 2015, acht Arbeitsgruppen mit einem thematischen Schwerpunkt zu bilden, deren Führung jeweils einem institutionellen Tandem oblag: Wirtschaft - Beschäftigung - Berufsausbildung (CD 57 - CRL - Chambre Régionale de Commerce et d'Industrie); Hochschule und Forschung (CRL Université de Lorraine); Erlernen der Sprache des Nachbarn und Schüleraustausch (CD 54 et 57 - Rectorat de l'Académie Nancy-Metz); Kultur und Tourismus (Communauté Urbaine du Grand Nancy - Communauté d'Agglomération de Metz Métropole/Sillon); Öffentlichkeitsarbeit (CRL et CESEL); Organisation des institutionellen und des Verwaltungsaustauschs (CD 54 Centre National de la Fonction Publique Territoriale); Mobilität (CRL CD 57); Bürgeraustausch (Metz - Nancy). Alle Gruppen hatten ein und dieselbe Doppelaufgabe: Für ihren jeweiligen Themenbereich eine Bestandsaufnahme der schon existierenden deutsch-französischen Aktivitäten vorzunehmen und auf dieser Basis Vorschläge für deren Weiterentwicklung zu machen. Dank der Arbeit dieser acht Arbeitsgruppen legte die politische Pilotgruppe schließlich im Sommer 2015 ihren Abschlussbericht vor, der Impulse für eine Intensivierung der Zusammenarbeit mit Deutschland in drei Etappen - kurz-, mittel- und langfristig - umfasst.

Im Hinblick auf die jeweiligen Zeithorizonte wurde als das generelle Ziel für alle Bereiche formuliert: 2015/16 die Kommunikation und die Koordination des Bestehenden zu verstärken; 2016/17 nicht nur Bestehendes, sondern auch neue Projekte voranzutreiben und schließlich ab 2018 die Zusammenarbeit tatsächlich sukzessive zu vertiefen. Um diese Ziele zu erreichen, sollen die ehemaligen acht Arbeitsgruppen in ,groupes opérationnels“" ('operationelle Gruppen') umgewandelt werden, die ihre Arbeit unter der Leitung der auch weiter bestehenden politischen Pilotgruppe durchführen. ${ }^{15}$ Diese wiederum soll ad hoc deutsche Partner und Spezialisten für einzelne Themenbereiche kooptieren. Klar ist dabei, dass sich ähnlich der Frankreichstrategie des Saarlandes auch die Deutschlandstrategie Lothringens nicht auf die regionale deutsch-französische Zusammenarbeit beschränken will, sondern das Partnerland insgesamt in den Blick nimmt. Zudem versteht auch sie sich als Beitrag zur Vertiefung der europäischen Einigung. Angesichts des spezifischen Kontextes der französischen Territorialreform ist schließlich die Hoffnung, dass die lothringische Deutschlandstrategie geradezu strukturbildend für die neue Großregion Alsace - Champagne - Ardenne - Lorraine (ACAL) werden könnte. Für diese sollte die transregionale und europäische Zusammenarbeit aufgrund ihrer geographischen Lage und ihrer Grenzen mit vier europäischen Ländern eine Selbstverständlichkeit, ja eine Notwendigkeit sein.

Der 'Aktionsplan', den sich die politisch Verantwortlichen im Sommer 2015 vorgegeben haben, wird interessanterweise jenseits seiner allgemeinen 
Ankündigungen auch schon recht konkret: ${ }^{16}$ So erscheint 2015/16 als eine ,Scharnierphase', die dank dreier Aktionen die Bedeutung der neuen Deutschlandstrategie verdeutlichen soll: erstens der Unterzeichnung einer gemeinsamen Konvention der Gebietskörperschaften mit dem Erziehungsministerium, um das Erlernen der deutschen Sprache weiterzuentwickeln; zweitens die Bildung saarländisch-lothringischer Cluster im Bereich der Automobilindustrie und der silver economy; drittens eine Kommunikationsoffensive, um die breite Öffentlichkeit von der Bedeutung der Beziehungen mit Deutschland zu überzeugen. Darüber hinaus enthält der Abschlussbericht vom Sommer 2015 eine Fülle von Vorschlägen aus den verschiedenen Arbeitsgruppen, jeweils gegliedert in die drei genannten zeitlichen Etappen. Alles in allem haben die acht Arbeitsgruppen der Entwicklung einer lothringischen Deutschlandstrategie sicher wertvolle Impulse vermittelt und zudem vielversprechende Perspektiven für eine Verzahnung dieses Projekts mit der saarländischen Frankreichstrategie aufgezeigt. Es bleibt nun abzuwarten, ob die Vorschläge auch in die Tat umgesetzt werden können.

\section{Perspektiven der saarländischen Frankreichstrategie und der lothringischen Deutschlandstrategie}

Im Laufe der letzten gut drei Jahre ist es der saarländischen Landesregierung gelungen, die eigene Frankreichstrategie diskursiv fest zu verankern, sogar über die regionale Ebene hinaus. Insofern hat der sogenannte zweite Teil der Frankreichstrategie, die „Kommunikationsstrategie (Vermarktung der saarländischen Frankreich-Kompetenz nach außen, d. h. nach Frankreich und nach Deutschland)"17 durchaus schon Erfolge gezeigt. Dank ihr hat das Saarland deutlich an Sichtbarkeit gewonnen, ein Umstand, der angesichts seiner demographischen und wirtschaftlichen Probleme nicht gering zu schätzen ist. Gleichzeitig sichert dies dem Bundesland auch einen deutlichen Vorsprung vor seiner französischen Partnerregion, die sicher zu zögerlich die Gunst der Stunde genutzt hat, um eine spiegelbildliche Strategie zu gestalten. Immerhin liegt nun auch hier eine sehr nützliche Bestandsaufnahme des eigenen deutsch-französischen Kapitals und dessen zu entwickelnden Potentials vor. Sinnvoll bleibt zweifellos auch für beide Regionen, ihre jeweilige Strategie auszubauen und sich hierbei abzustimmen, denn letztlich leiden beide unter denselben Problemen: einer fehlenden Reputation auf nationaler und internationaler Ebene und ungelösten wirtschaftlichen Problemen. Gleichzeitig

16 Vgl. Stratégie Allemagne de la Lorraine, S. 42-44.

17 Staatskanzlei des Saarlandes: Eckpunkte einer Frankreichstrategie für das Saarland, 2014, http:// www.saarland.de/dokumente/res_stk/D_Eckpunkte_Frankreich-Strategie_210114.pdf (14.04.2016), S. 3. 
ergänzen sich gerade im wirtschaftlichen Bereich saarländische und lothringische Interessen: Dem demographisch bedingten Arbeitskräftemangel im Saarland steht ein Arbeitskräfteüberhang in Lothringen gegenüber.

Nicht nur die bereits zitierten aktuellen Zahlen, sondern auch die Projektionen für die kommenden zehn Jahre sind hier eindeutig: Das französische Statistik- und Wirtschaftsforschungsinstitut INSEE prognostiziert für den Zeitraum bis 2025 einen jährlichen Anstieg der erwerbstätigen französischen Bevölkerung von 110000 Personen. ${ }^{18}$ Für Deutschland wird hingegen von einem jährlichen Rückgang um 100000 Personen ausgegangen. Hier, so ist die lothringische Hoffnung, könnten sich besondere Möglichkeiten für die eigene Region ergeben, zumal die Vorhersagen auch für den unmittelbaren saarländischen Nachbarn einen Bevölkerungsrückgang auf unter 900000 bis 2030 vorhersagen. Bereits jetzt fehlen der saarländischen Industrie Facharbeiter. Insofern ist das Rahmenabkommen zur grenzüberschreitenden Berufsausbildung, das Lothringen und das Saarland im Juni 2014 unterzeichnet haben, ein Schritt in die richtige Richtung. ${ }^{19}$ Auch ist es offensichtlich, dass beide Regionen aufgrund ihrer Grenzlage eine Pilotrolle für die deutschfranzösischen Beziehungen spielen könnten, und zudem im Bereich der Automobilindustrie, aber auch der sogenannten silver economy gemeinsam lohnende Projekte entwickeln könnten.

Indes sind auch die Probleme bei der Umsetzung der beiden ambitionierten Großprojekte ebenso unübersehbar und auch sie betreffen zumindest teilweise das Saarland wie Lothringen gleichermaßen: Erstens lässt die konkrete Umsetzung der Frankreichstrategie, also die sogenannte „Binnenstrategie“, die „Stärkung der Frankreichkompetenz im Land“" 20 und insbesondere deren Kernelement, die Zweisprachigkeit, noch auf sich warten. Natürlich ist hier Geduld gefordert, schließlich hat sich die Landesregierung ja bewusst 30 Jahre Zeit gegeben. Indes muss man realistisch sein: Damit das Zieldatum 2043 eingehalten werden kann und die Frankreichstrategie nicht im Zuge politischer Wechsel in den nächsten Jahren und Jahrzehnten grundsätzlich wieder in Frage gestellt wird, muss sie bald deutliche Fortschritte machen, sozusagen den point of no return überschreiten und eine Pfadabhängigkeit kreieren, um damit unumkehrbar und verbindlich auch für die zukünftige

18 Vgl. Stratégie Allemagne de la Lorraine, S. 7; vgl. Sievert, Stephan/Klingholz, Reiner: Ungleiche Nachbarn. Die demografische Entwicklung in Deutschland und Frankreich verläuft gegensätzlich - mit enormen Langzeitfolgen; Berlin: Berlin-Institut für Bevölkerung und Entwicklung, 2009, http: //www.berlin-institut.org/fileadmin/user_upload/Veroeffentlichungen/Frankreich/ Ungleiche_Nachbarn_online_NEU.pdf (14.04.2016).

19 Vgl. Abkommen über die grenzüberschreitende Berufsausbildung Saarland-Lothringen/ Accord relatif à l'apprentissage transfrontalier Sarre-Lorraine, http://www.saarland.de/ dokumente/thema_europa/gufb_Abkommen.pdf (14.04.2016).

20 Staatskanzlei des Saarlandes: Eckpunkte einer Frankreichstrategie, S. 3. 
Landespolitik zu werden. Diese Ambition stößt sich indes an Haushaltszwängen, unter denen auch diverse frankreichspezifische Aktivitäten leiden. Hier müsste zweitens eine klare Prioritätensetzung erfolgen. Drittens sollte noch mehr als bisher der Kontakt mit der Zivilgesellschaft und ein wirklicher Erfahrungsaustausch mit den frankreichspezifischen Akteuren im Land, aber auch darüber hinaus, erfolgen, um die Frankreichstrategie noch stärker innergesellschaftlich zu verankern, aber auch um weitere konkrete Anregungen und ggf. sogar Lösungsvorschläge für bestehende Probleme zu erhalten. Die Expertise ist vorhanden, sie müsste abgerufen werden und gleichzeitig müsste dabei offen, wohl auch offener als bisher, über noch bestehende Defizite geredet werden. Die bereits eingeführte Veranstaltungsform des ,Runden Tischs', der sich zuletzt am 17. Januar 2017 in der Staatskanzlei getroffen hat ${ }^{21}$ ist sicher eine lobenswerte Idee. Indes sollten die Veranstaltungen weniger grande messe, sondern vielmehr echte Arbeitstreffen mit offenem, wenn nötig auch kontroversem Meinungsaustausch sein.

Fortschritte der saarländischen Frankreichstrategie könnten gleichzeitig die lothringische Deutschlandstrategie vorantreiben - und umgekehrt. Indes ist die Herausforderung auf französischer Seite noch größer: Erstens ist Lothringen seit dem 1. Januar 2016 nur noch Teil der schon erwähnten größeren Region Grand Est. ${ }^{22}$ Damit stellt sich die Frage nach der Kompetenzverteilung und folglich nach der zukünftigen Rolle der lothringischen Teilregion im neuen Gesamtgefüge. Mit dem Elsass gibt es nun einen weiteren, deutschlandpolitisch sehr starken Akteur, der regional indes eher nach BadenWürttemberg ausgerichtet ist. Champagne-Ardenne wiederum ist nicht nur geographisch weiter von Deutschland entfernt. Insofern ist die Deutschlandorientierung innerhalb des Grand Est nicht überall gleich stark ausgeprägt. Das Interesse Lothringens müsste es dennoch auf jeden Fall sein, weiter von seiner spezifischen Deutschlandkompetenz zu profitieren, sei es unter Beibehaltung seiner eigenen entsprechenden Strategie oder nun im Rahmen einer gemeinsamen Strategie der neuen französischen Großregion, deren unmittelbare regionale Partner in Deutschland dann sowohl das Saarland als auch Baden-Württemberg wären. Bislang sprechen hierfür aus lothringischer Perspektive, wie schon erwähnt, starke arbeitsmarktpolitische Erwägungen. Hier aber muss eine zweite Einschränkung gemacht werden: Der aktuelle Zuzug von Hunderttausenden von Flüchtlingen könnte zumindest langfristig Deutschlands demographische Probleme lösen und eine weitere gezielte Anwerbung von Arbeitskräften aus dem EU-Ausland, darunter auch aus Frankreich,

21 Vgl. Saarnews: Runder Tisch Frankreichs, 18.01.2017, http://saarnews.com/index.php/ 2017/01/18/runder-tisch-frankreich (31.07.2017).

22 Grillmeyer, Dominik: Territorialreform in Frankreich. Ambitionierter Start, Schwächen im Abschluss (= DGAPAnalyse 13, 28.10.2015), https://dgap.org/de/think-tank/ publikationen/dgapanalyse/territorialreform-frankreich-0 (13.04.2016). 
obsolet werden lassen. Allerdings ändert dieser Einwand zumindest nichts an der kurz- und vielleicht sogar mittelfristigen Perspektive. Zudem kann sich auch eine nur vorübergehende Beschäftigung beim deutschen Nachbarn als ein Turbo für eine weitere berufliche Laufbahn in Frankreich erweisen. In diesem Zusammenhang ist sehr bedauerlich, dass bisher nur wenige junge Deutsche und Franzosen erkennen, welche arbeitsmarktpolitischen Vorteile ihnen das Erlernen der Sprache des Anderen, ergänzend natürlich zum Englischen, bietet, gerade weil diese Option aktuell in beiden Ländern nur von einer kleinen Minderheit gewählt wird, die den tatsächlichen Bedarf an gut ausgebildeten und sprachlich sicheren, aber auch interkulturell erfahrenen Nachwuchskräften in Kultureinrichtungen, öffentlicher Verwaltung und Unternehmen nicht stillen kann.

Drittens ist im Hinblick auf die Zukunftsaussichten der beiden Strategien daran zu erinnern, dass beide Regionen - das Saarland wie der Grand Est für die Verankerung der deutsch-französischen Beziehungen eine wichtige Rolle spielen könnten. Seit gut anderthalb Jahrzehnten erleben wir national wie international einen umfassenden Generationenwechsel: Jene Altersgruppe, die den Zweiten Weltkrieg und seine Folgen noch selbst erlebt hat und der die Aussöhnung mit den Nachbarn daher nach 1945 eine Herzensangelegenheit war, macht endgültig einer neuen Platz, für die „Europa [...] nicht mehr eine Sache des Instinkts oder des Herzens [ist], es ist eine Sache der Vernunft “23. Gleichzeitig ist die deutsch-französische Aussöhnung zum Opfer ihres eigenen Erfolges geworden. Das beeindruckend hohe Maß an Austausch, Kenntnis und Zusammenarbeit wird von einer jüngeren Generation nicht nur für selbstverständlich erachtet, sondern hat die deutschfranzösischen Beziehungen geradezu banalisiert. Dies erklärt zweifellos auch die Abnahme der Deutschlerner in Frankreich und jene der deutschen Schüler, die Französisch lernen wollen. Hieraus rührt die bekannte Klage Pierre Noras von 2012, Deutsche und Franzosen hätten sich ,auseinandergelebt“"24, oder Peter Sloterdijks Feststellung, das deutsch-französische Verhältnis sei „günstigenfalls als das einer wohlwollenden gegenseitigen NichtBeachtung oder benignen Entfremdung ${ }^{\text {“25 }}$ zu bezeichnen. In der Tat drohen

23 Vernet, Daniel: Ungewissheiten in der Europa-Politik. Neue deutsch-französische Entscheidungsträger, in: Internationale Politik 9 (1998), S. 1-6, hier S. 3; vgl. Baasner, Fritz: Der Generationswechsel nach der Aussöhnung, in: Dokumente. Zeitschrift für den deutsch-französischen Dialog 3 (2006), S. 30-33.

24 Guez, Olivier: Man hat sich auseinandergelebt. Lässt sich dem recht bescheiden gewordenen kulturellen Austausch zwischen Frankreich und Deutschland aufhelfen? Der prominente Historiker Pierre Nora bleibt skeptisch, in: Frankfurter Allgemeine Zeitung, 16.02.2012, http://www.faz.net/aktuell/feuilleton/deutsch-franzoesisches-verhaeltnis-man-hat-sichauseinandergelebt-11651980.html (13.04.2016).

25 Sloterdijk, Peter: Theorie der Nachkriegszeiten. Bemerkungen zu den deutsch-französischen Beziebungen seit 1945, Frankfurt/M.: Suhrkamp, 2008, S. 9. 
die eingetretenen Automatismen und Rituale Deutsche und Franzosen dazu zu verleiten, sich allzu sehr in der Sicherheit einer endgültigen Pfadabhängigkeit und damit Unumkehrbarkeit des Erreichten zu wiegen.

Indes darf auch nicht unterschätzt werden, wie stark sich beide Völker mittlerweile ,zusammengelebt“ 26 haben und wie sehr gerade diese Sonderbeziehung immer noch eine vielversprechende Zukunft haben kann. Das integrierte Europa ist dank der beiden Länder und ihrer Zusammenarbeit in den 1950er Jahren überhaupt erst entstanden. ${ }^{27}$ Auch wenn es im Europa der 28 von heute - oder nach dem vollzogenen ,Brexit' dann der 27 - andere Gewichtungen gibt als im einstigen Europa der 6, so gilt doch weiterhin die Regel: Ohne ein Einvernehmen von Deutschland und Frankreich ist die Europäische Union nicht handlungsfähig. ${ }^{28}$ Eine andauernd effektive Kooperation setzte allerdings voraus, dass die Eliten wie die Masse der Bevölkerung beider Länder sich auch zukünftig für die deutsch-französischen Beziehungen interessieren: Hierzu ihren Beitrag zu leisten, dazu sind insbesondere die Grenzregionen aufgerufen. Das Saarland und Lothringen bzw. der französische Grand Est könnten hierbei eine prominente Pilotrolle spielen. Insofern ist der politische Voluntarismus, wie er sich in den spiegelbildlichen Strategieprojekten der beiden Regionen offenbart, auf jeden Fall zu begrüßen und ihm Nachhaltigkeit zu wünschen, gleich ob er am Ende seine jeweiligen Maximalziele erreicht oder nicht.

26 Teufel, Erwin/Baasner, Fritz: Deutsch-Französische Verständigung. Wir haben uns zusammengelebt. Pessimismus ist hier fehl am Platz: Deutsche und Franzosen verstehen sich auch in einer zunehmend komplexen Welt noch gut. Eine Antwort auf Pierre Nora., in: Frankfurter Allgemeine Zeitung, 26.03.2012, http://www.faz.net/aktuell/feuilleton/deutschfranzoesische-verstaendigung-wir-haben-uns-zusammengelebt-11698459.html (05.10.2016).

$27 \mathrm{Zu}$ den deutsch-französischen Beziehungen nach 1945 im europäischen Kontext vgl. Defrance, Corine/Pfeil, Ulrich: Eine Nachkeriegsgeschichte in Europa 1949 bis 1963, Darmstadt: WBG, 2011; Miard-Delacroix, Hélène: Im Zeichen der europä̈schen Einigung. 1963 bis in die Gegenwart, Darmstadt: WBG, 2011; Seidendorf, Stefan (Hg.): Deutsch-Französische Beriehungen als Modellbankasten? Zur Übertragbarkeit von Aussöbnung und strukturierter Zusammenarbeit, Baden-Baden: Nomos, 2012; Marcowitz, Reiner/Miard-Delacroix, Hélène (Hg.): 50 ans de relations franco-allemandes, Paris: Nouveau monde éditions, 2013.

28 Vgl. Martens, Stephan/Gustin, Philippe: Relancer le moteur de l'Europe, Paris: Lemieux Editeur, 2016. 


\section{Literaturverzeichnis}

Abkommen über die grenzüberschreitende Berufsausbildung Saarland-Lothringen/Accord relatif à l'apprentissage transfrontalier Sarre-Lorraine, http://www.saarland.de/dokumente/ thema_europa/gufb_Abkommen.pdf (14.04.2016).

Baasner, Fritz: Der Generationswechsel nach der Aussöhnung, in: Dokumente. Zeitscbrift für den deutsch-französischen Dialog 3 (2006), S. 30-33.

Bastien, René: Histoire de la Lorraine, Metz: Editions Serpenoise, 1993.

Conseil Economique, Social et Environnemental de Lorraine (CESEL): L'Allemagne, partenaire incontournable de la Lorraine. Projet. Séance Plénière du 18 octobre 2011, http://www. ceselorraine.eu/UserFiles/File/ressources/rapports/2011/2011-cesel-rapport-allemagne. $\operatorname{pdf}(14.04 .2016)$.

Conseil Economique, Social et Environnemental de Lorraine (CESEL): Avis du CESEL sur la Stratégie France de la Sarre. Une opportunité à saisir, une obligation d'agir. Avis adopté à l'unanimité en Séance Plénière du 16 mai 2014, http://www.ceselorraine.eu/img_base/ ged_v2/278/2014-cesel-avis-strategie-france-sarre.pdf. (14.04.2016).

Conseil Economique, Social et Environnemental de Lorraine (CESEL): A la Une. Stratégie France: le CESEL présente 8 pistes d'actions côté lorrain, 20.05.2014, http://www. ceselorraine.eu/fr/strategie-france-le-cesel-presente-8-pistes-d-actions-cote-lorrain/a-laune-du-cese-lorraine.html (14.04.2016).

Defrance, Corine/Pfeil, Ulrich: Eine Nachkriegsgeschichte in Europa 1949 bis 1963, Darmstadt: WBG, 2011.

Delorme, Florian: La bataille des langues en Europe (2/4) - De l'école au travail, enjeux linguistiques en zones transfrontalières, in: France Culture Radio, 18.03.2014, http://www. franceculture.fr/emissions/culturesmonde/la-bataille-des-langues-en-europe-24-delecole-au-travail-enjeux (14.04.2016).

Grillmeyer, Dominik: Territorialreform in Frankreich. Ambitionierter Start, Schwächen im Abschluss (= DGAPAnalyse 13, 28.10.2015), https://dgap.org/de/think-tank/publikationen/ dgapanalyse/territorialreform-frankreich-0 (13.04.2016).

Guez, Olivier: Man hat sich auseinandergelebt. Lässt sich dem recht bescheiden gewordenen kulturellen Austausch zwischen Frankreich und Deutschland aufhelfen? Der prominente Historiker Pierre Nora bleibt skeptisch, in: Frankfurter Allgemeine Zeitung, 16.02.2012, http://www.faz.net/aktuell/feuilleton/deutsch-franzoesisches-verhaeltnis-man-hat-sichauseinandergelebt-11651980.html (13.04.2016).

Knoepffler, Christian: Frankreich über alles, in: Le Républicain Lorrain, 05.02.2014, S. 7.

Marcowitz, Reiner/Miard-Delacroix, Hélène (Hg.): 50 ans de relations franco-allemandes, Paris: Nouveau monde éditions, 2013.

Martens, Stephan/Gustin, Philippe: Relancer le moteur de l'Europe, Paris: Lemieux Editeur, 2016.

Miard-Delacroix, Hélène: Im Zeichen der europäischen Einigung. 1963 bis in die Gegenwart, Darmstadt: WBG, 2011.

O. A.: Pour survivre, la Sarre parie sur le parler français, in : Le Télégramme, 03.03.2014, S. 1.

Parisse, Michel (Hg.): Lotbringen. Geschichte eines Gren₹landes, Saarbrücken: Saarbrücker Druckerei u. Verl., 1983.

Saarnews: Runder Tisch Frankreichs, 18.01.2017, http://saarnews.com/index.php/2017/01/ 18/runder-tisch-frankreich (31.07.2017).

Seidendorf, Stefan (Hg.): Deutsch-Franఇösische Beriebungen als Modellbaukasten? Zur Übertragbarkeit von Aussöbnung und strukturierter Zusammenarbeit, Baden-Baden: Nomos, 2012. 
Sievert, Stephan/Klingholz, Reiner: Ungleiche Nacbbarn. Die demografische Entwicklung in Deutschland und Frankereich verläuft gegensätəlich - mit enormen Langzeitfolgen, Berlin: Berlin-Institut für Bevölkerung und Entwicklung, 2009, http://www.berlin-institut.org/fileadmin/user_ upload/Veroeffentlichungen/Frankreich/Ungleiche_Nachbarn_online_NEU.pdf (14.04.2016).

Sloterdijk, Peter: Theorie der Nachkriegszeiten. Bemerkungen zu den deutsch-französischen Beziehungen seit 1945, Frankfurt/M.: Suhrkamp, 2008.

Staatskanzlei des Saarlandes: Eckpunkte einer Frankreichstrategie für das Saarland, 2014, http:// www.saarland.de/dokumente/res_stk/D_Eckpunkte_Frankreich-Strategie_210114.pdf (14.04.2016).

Stellungnahme der Universität Lothringen zur Frankreichstrategie des Saarlandes vom 02.06.2014, Kopie im persönlichen Besitz des Autors.

Stratégie Allemagne de la Lorraine. Rapport final. Synthèse et propositions juin 2015, 2015, http:/ / metz.fr/ pages/conseil_municipal/seances/cm151029/doc/5_d1445930773387.pdf (14.04.2016).

Teufel, Erwin/Baasner, Fritz: Deutsch-Französische Verständigung. Wir haben uns zusammengelebt. Pessimismus ist hier fehl am Platz: Deutsche und Franzosen verstehen sich auch in einer zunehmend komplexen Welt noch gut. Eine Antwort auf Pierre Nora., in: Frankfurter Allgemeine Zeitung, 26.03.2012, http://www.faz.net/aktuell/feuilleton/ deutsch-franzoesische-verstaendigung-wir-haben-uns-zusammengelebt-11698459.html (05.10.2016).

Vernet, Daniel: Ungewissheiten in der Europa-Politik. Neue deutsch-französische Entscheidungsträger, in: Internationale Politik 9 (1998), S. 1-6. 
Umsetzung - Perspektiven aus Wissenschaft und Politik La mise en application - Perspectives scientifiques et politiques 
